\title{
On a Cournot Game with Bounded Rational Players, Convex, Log-Linear Demand and Consumer Surplus
}

\section{Georges Sarafopoulos and Kosmas Papadopoulos}

Department of Economics, Democritus University of Thrace, Komotini, 69100, Greece

\section{Abstract}

Based on the Cournot oligopoly game and the nonlinear dynamics theory, we study the behavior of semi-public enterprises by considering corporate social responsibility into their objectives. The model that is established is a dynamical Cournot-type duopoly model with bounded rationality containing the consumer surplus. We suppose quadratic cost function and a convex, log-linear demand function. The game is modeled with a system of two difference equations. Existence and stability of equilibriums of this system are studied. More complex chaotic and unpredictable trajectories are resulted studying this discrete dynamical system. The complex dynamics of the system are

Corresponding Author: Georges Sarafopoulos gsarafop@econ.duth.gr

Received: 17 November 2019 Accepted: 6 January 2019 Published: 12 January 2020

Publishing services provided by Knowledge E

(c) Georges Sarafopoulos and Kosmas Papadopoulos. This article is distributed under the terms of the Creative Commons Attribution License, which permits unrestricted use and redistribution provided that the original author and source are credited.

Selection and Peer-review under the responsibility of the EBEEC Conference Committee.

\section{G OPEN ACCESS} demonstrated numerically via computing Lyapunov numbers, sensitivity dependence on initial conditions, and bifurcation diagrams.

Keywords: Cournot duopoly game; Discrete dynamical system; Homogeneous expectations; Stability; Chaotic Behavior; Consumer Surplus.

\section{Introduction}

Oligopoly is a market structure between monopoly and perfect competition, where there are only a small number of firms that produce homogeneous products. The dynamic of an oligopoly game is more complex because firms must consider not only the behaviors of the consumers, but also the reactions of the competitors i.e. they form expectations concerning how their rivals will act at every next time period. Augustin Cournot, in 1838 introduced the first formal theory of oligopoly. He treated the case with naive expectations. In this case, at every step each player (firm) assumes the last values that were taken by the competitors without estimation of their future reactions.

Expectations play an important role in modelling economic phenomena. A producer can choose his expectations rules of many available techniques to adjust his production outputs trying to maximize his utility (profit) functions. In this paper the dynamics of a duopoly model where each firm behaves with heterogeneous expectations strategies are studied. Also, a duopoly model where each player forms a strategy in order to 
compute his expected output is considered. Each player adjusts his outputs towards the profit maximizing amount as target by using his expectations rule. Some authors considered duopolies with homogeneous expectations and found a variety of complex dynamics in their games, such as appearance of strange attractors (Agiza [1], Agiza et al. [4], Agliari et al., [5],[6], Bischi, Kopel [10], Kopel [20], Puu [25], Sarafopoulos,[27]). Also models with heterogeneous agents were studied (Agiza, Elsadany [2], [3], Agiza et al. [4], Den Haan [14], Fanti, Gori [17], Tramontana [29], Zhang [32]).

In the real market producers do not know the entire demand function, though it is possible that they have a perfect knowledge of technology, represented by the cost function. Hence, it is more likely that firms employ some local estimate of the demand. This issue has been previously analyzed by Baumol and Quandt [9], Puu [24], Naimzada and Ricchiuti [22], Askar [7], Askar [8]. Efforts have been made to model bounded rationality to different economic areas: oligopoly games (Agiza, Elsadany [3], Bischi et al [12], Zhang et al. [32]); financial markets (Hommes [19]); macroeconomic model such as multiplier-accelerator framework (Westerhoff [30]). In particular, difference equations have been employed extensively to represent these economic phenomenona (Elaydi [15], Sedaghat [28]). Bounded rational players (firms) update their production strategies based on discrete time periods and by using a local estimate of the marginal profit. With such a local adjustment mechanism, the players are not requested to have a complete knowledge of the demand and the cost functions (Agiza, Elsadany [2], Naimzada, Sbragia [23], Zhang et al [31], Askar [8]). All they need to know is if the market responses to small production changes by an estimate of the marginal profit.

All of the above discussions are mainly based on private enterprises, which pursuit the maximization of their own profits. However, there are many firms with different ownership structures. A publicly-owned firm tends to maximize the social welfare, a partially publicly-owned firms tend to maximize the weighted average of the social welfare and its own profit. (Elsadany, Awad [16]).

The present study is a partial theoretical approach to our main ongoing research objective, which is to quantify and study an oligopoly of the Greek market and extends Askar [8] and Sarafopoulos [27]. We investigate the dynamics of a nonlinear discretetime duopoly game, where the players are bounded rational and they have homogeneous expectations. We assume that the two bounded rational players not only pursue profit maximization but also take consumer surplus into account, so the utilities of both players are the combination of their profits and the consumer surplus. Existence and stability of equilibrium of this system are studied. We show that the model gives more complex chaotic and unpredictable trajectories as a consequence of change in 
the speed of adjustment of the players and the new parameter $\mu$ that is imported in players' utilities which reveals the percentage of the consumer surplus they take into account. Moreover, from a mathematical point of view, we show that the destabilization of the fixed point can occur through a flip bifurcation and also that a cascade of flip bifurcations may lead to periodic cycles and deterministic chaos.

The paper is organized as follows: In Section 2, the construction of the duopoly game is made using log-linear demand and quadratic cost function. The utilities of two players are exported including a percentage of the consumer surplus and the discrete dynamical system of this game is constructed. The dynamics of this game with homogeneous expectations is analyzed. The existence and local stability of the equilibrium points are analyzed. In Section 3 numerical simulations are used to show complex dynamics via computing Lyapunov numbers, and sensitive dependence on initial conditions.

\section{The Game}

\subsection{The construction of the game}

In oligopoly game players can choose simple extension rules such as naïve or complicated as adaptive expectations and bounded rationality. The players can use the same strategy (homogeneous expectations) or can use different strategy (heterogeneous expectations). In this study we consider homogeneous players such that each player thinks with the same strategy to maximize his output (bounded rational player). Let us consider a market where two firms produce homogeneous commodities. Production decisions are taken at discrete time periods $(t=0,1,2, \ldots)$ We consider a simple Cournottype duopoly market where firms (players) produce the same good and offer them at discrete-time periods on a common market. At each period t, every firm must form an expectation of the rival's output in the next time period in order to determine the corresponding profit-maximization quantities for period $t+1$. The different technique in this study is that both players' utility function contains a percentage of the consumer surplus. We suppose that $q_{1}, q_{2}$ are the production quantities of each firm, then the inverse demand function (as a function of quantities) is given by the following equation:

$$
p=\alpha-b \cdot \ln \left(q_{1}+q_{2}\right)
$$


where $p$ is the price of their product and $\alpha$ is a positive parameter which expresses the market size. So, for the two players it means that the direct demand function is exponential:

$$
Q=q_{1}+q_{2}=e^{\frac{\alpha-p}{b}}
$$

Thus it is convex and log-linear in the price. A function is log-linear if and only if its logarithm is linear. The marketing interpretation of log-linearity is that the rate of growth of the quantity is constant. We suppose that the cost functions of the players are quadratic:

$$
C_{1}\left(q_{1}\right)=c \cdot q_{1}^{2} \text { and } C_{2}\left(q_{2}\right)=c \cdot q_{2}^{2} \text {, where } c>0
$$

With these assumptions the profits of the firms are given by the following equations:

$$
\pi_{1}\left(q_{1}, q_{2}\right)=p_{1} \cdot q_{1}-C_{1}\left(q_{1}\right)=\left[\alpha-b \cdot \ln \left(q_{1}+q_{2}\right)\right] \cdot q_{1}-c \cdot q_{1}^{2}
$$

and

$$
\pi_{2}\left(q_{1}, q_{2}\right)=p_{2} \cdot q_{2}-C_{2}\left(q_{2}\right)=\left[\alpha-b \cdot \ln \left(q_{1}+q_{2}\right)\right] \cdot q_{2}-c \cdot q_{2}^{2}
$$

Then, their marginal profits at the point of the strategy space are given by:

$$
\frac{\partial \pi_{1}}{\partial q_{1}}=\alpha-b \cdot \ln \left(q_{1}+q_{2}\right)-\left(2 c+\frac{b}{q_{1}+q_{2}}\right) \cdot q_{1}
$$

and

$$
\frac{\partial \pi_{2}}{\partial q_{2}}=\alpha-b \cdot \ln \left(q_{1}+q_{2}\right)-\left(2 c+\frac{b}{q_{1}+q_{2}}\right) \cdot q_{2}
$$

As it is noticed above the players of this game not only pursue their own profits but also take corporate social responsibility into account. Some empirical studies have shown how introducing corporate social responsibility affects firm's performance, where we interpret the corporate social responsibility as either consumer surplus CS or social welfare SW. In this game we take account of the CS. Now, the CS can be written as:

$$
C S=\int_{p}^{+\infty} e^{\frac{\alpha-p}{b}} d p=\lim _{n \rightarrow \infty}\left(\int_{p}^{n} e^{\frac{\alpha-p}{b}} d p\right)=b \cdot\left(q_{1}+q_{2}\right)
$$


As it is said above, each player cares about the maximization of a utility function that contains his profit function and a percentage of the consumer surplus. This utility function $U$ is described by the following equation:

$$
U_{i}\left(q_{i}, q_{j}\right)=(1-\mu) \cdot \pi_{i}+\mu \cdot C S
$$

where $\mu \in[0,1]$ is the percentage of the CS is taken into account by the player i. So, for the players of this game it means that:

$$
U_{1}\left(q_{1}, q_{2}\right)=(1-\mu) \cdot \pi_{1}+\mu \cdot C S
$$

and

$$
U_{2}\left(q_{1}, q_{2}\right)=(1-\mu) \cdot \pi_{2}+\mu \cdot C S
$$

Also, the marginal utilities are given by the following equations:

$$
\begin{gathered}
\frac{\partial U_{1}}{\partial q_{1}}=(1-\mu) \cdot \frac{\partial \pi_{1}}{\partial q_{1}}+\mu \cdot \frac{\partial C S}{\partial q_{1}} \Leftrightarrow \\
\frac{\partial U_{1}}{\partial q_{1}}=(1-\mu) \cdot\left[\alpha-b \cdot \ln \left(q_{1}+q_{2}\right)-\left(2 c+\frac{b}{q_{1}+q_{2}}\right) \cdot q_{1}\right]+\mu \cdot b
\end{gathered}
$$

And

$$
\begin{gathered}
\frac{\partial U_{2}}{\partial q_{2}}=(1-\mu) \cdot \frac{\partial \pi_{2}}{\partial q_{2}}+\mu \cdot \frac{\partial C S}{\partial q_{2}} \Leftrightarrow \\
\frac{\partial U_{2}}{\partial q_{2}}=(1-\mu) \cdot\left[\alpha-b \cdot \ln \left(q_{1}+q_{2}\right)-\left(2 c+\frac{b}{q_{1}+q_{2}}\right) \cdot q_{2}\right]+\mu \cdot b
\end{gathered}
$$

Both players follow the same strategy to decide their production quantities (homogeneous players) and they are characterized as bounded rational players. According to the existing literature it means that they decide their productions following a mechanism that is described by the equation:

$$
\frac{q_{i}(t+1)-q_{i}(t)}{q_{i}(t)}=k \cdot \frac{\partial U_{i}}{\partial q_{i}}, \quad i=1,2
$$


Through this mechanism each player increases his level of adaptation when his marginal utility is positive or decreases his level when his marginal utility is negative, where $\mathrm{k}$ is the speed of adjustment of two players, it is a positive parameter $(k>0)$, which gives the extend variation production of the firm i following a given utility signal.

The dynamical system of the players is described by:

$$
\left\{\begin{array}{l}
q_{1}(t+1)=q_{1}(t)+k \cdot q_{1}(t) \cdot \frac{\partial U_{1}}{\partial q_{1}} \\
q_{2}(t+1)=q_{2}(t)+k \cdot q_{2}(t) \cdot \frac{\partial U_{2}}{\partial q_{2}}
\end{array}\right.
$$

We investigate the effect of the parameter $k$ (speed of adjustment) and the parameter $\mu$ on the dynamics of this system.

\subsection{Dynamical analysis}

\subsubsection{The equilibriums of the game}

The equilibrium positions are the nonnegative solutions of the algebraic system:

$$
\left\{\begin{array}{l}
q_{1}^{*} \cdot \frac{\partial U_{1}}{\partial q_{1}}=0 \\
q_{2}^{*} \cdot \frac{\partial U_{2}}{\partial q_{2}}=0
\end{array}\right.
$$

which obtained by setting: $q_{1}(t+1)=q_{1}(t)=q_{1}^{*}$ and $q_{2}(t+1)=q_{2}(t)=q_{2}^{*}$ in the dynamical system of $\mathrm{Eq}(13)$.

- If $q_{1}^{*}=q_{2}^{*}=0$, then the equilibrium position $E_{0}=(0,0)$ is rejected because $q_{1}+q_{2}>0$.

- If $q_{1}^{*}=0$ and $\frac{\partial U_{2}}{\partial q_{2}}=0$, then:

$$
b \cdot \ln q_{2}^{*}+2 c q_{2}^{*}+b-\alpha-\frac{\mu b}{1-\mu}=0
$$

We consider the function:

$$
h(x)=b \cdot \ln x+2 c x+b-\alpha-\frac{\mu b}{1-\mu}
$$


with $D_{h}=(0,+\infty)$ and $h^{\prime}(x)=\frac{b}{x}+2 c>0$, so the function $h(x) \nearrow$ for $x>0$ (monotonic function). Also, $0 \in h\left(D_{h}\right)=\mathbb{R}$ and because $\mathrm{h}$ is a monotonic function, it means that there is a unique root $x^{*}$ of $h(x)=0$ and the equilibrium position is $E_{1}=\left(0, y_{1}\right)$, where $y_{1}$ is the unique positive solution of Eq.(15).

- If $q_{2}^{*}=0$ and $\frac{\partial U_{1}}{\partial q_{1}}=0$, then:

$$
b \cdot \ln q_{1}^{*}+2 c q_{1}^{*}+b-\alpha-\frac{\mu b}{1-\mu}=0
$$

and the equilibrium position is the $E_{2}=\left(x_{2}, 0\right)$, where using the function $\mathrm{h}$ again the $x_{2}$ is the unique positive solution of Eq.(17).

- If $\frac{\partial U_{1}}{\partial q_{1}}=\frac{\partial U_{2}}{\partial q_{2}}=0$, it gives the system:

$$
\left\{\begin{array}{l}
(1-\mu) \cdot\left[\alpha-b \cdot \ln \left(q_{1}^{*}+q_{2}^{*}\right)-\left(2 c+\frac{b}{q_{1}^{*}+q_{2}^{*}}\right) \cdot q_{1}^{*}\right]+\mu \cdot b=0 \\
(1-\mu) \cdot\left[\alpha-b \cdot \ln \left(q_{1}^{*}+q_{2}^{*}\right)-\left(2 c+\frac{b}{q_{1}^{*}+q_{2}^{*}}\right) \cdot q_{2}^{*}\right]+\mu \cdot b=0
\end{array}\right.
$$

and also:

$$
\begin{aligned}
& \alpha-b \cdot \ln \left(q_{1}^{*}+q_{2}^{*}\right)-\left(2 c+\frac{b}{q_{1}^{*}+q_{2}^{*}}\right) \cdot q_{1}^{*}= \\
& \alpha-b \cdot \ln \left(q_{1}^{*}+q_{2}^{*}\right)-\left(2 c+\frac{b}{q_{1}^{*}+q_{2}^{*}}\right) \cdot q_{2}^{*} \Leftrightarrow \\
& q_{1}^{*}=q_{2}^{*}=q^{*}
\end{aligned}
$$

Now, form Eq.(18) with $q_{1}^{*}=q_{2}^{*}=q^{*}$ we obtain:

$$
\begin{gathered}
(1-\mu) \cdot\left[\alpha-b \cdot \ln \left(2 q^{*}\right)-\left(2 c+\frac{b}{2 q^{*}}\right) \cdot q^{*}\right]+\mu \cdot b=0 \Leftrightarrow \\
b \cdot \ln q^{*}+2 c q^{*}+b-\alpha-\frac{\mu b}{1-\mu}-\frac{b}{2}=0
\end{gathered}
$$

We consider the function $k(x)=h(x)+b \cdot \ln 2-\frac{b}{2}=h(x)+s$, where $s=b \cdot \ln 2-\frac{b}{2}$, with $D_{k}=D_{h}=(0,+\infty)$. The function $k(x)$ has also a unique positive solution $x^{*}=q^{*}$ and $E_{*}=\left(q^{*}, q^{*}\right)$ is the Nash equilibrium. 


\subsubsection{Stability of equilibriums}

To study the stability of game's equilibriums, the Jacobian matrix is used. The Jacobian matrix $J\left(q_{1}, q_{2}\right)$ along the variable strategy $\left(q_{1}, q_{2}\right)$ is:

$$
J\left(q_{1}, q_{2}\right)=\left[\begin{array}{ll}
f_{q_{1}} & f_{q_{2}} \\
g_{q_{1}} & g_{q_{2}}
\end{array}\right]
$$

where:

$$
f\left(q_{1}, q_{2}\right)=q_{1}+k \cdot q_{1} \cdot \frac{\partial U_{1}}{\partial q_{1}}
$$

and

$$
g\left(q_{1}, q_{2}\right)=q_{2}+k \cdot q_{2} \cdot \frac{\partial U_{2}}{\partial q_{2}}
$$

The Jacobian matrix becomes:

$$
J\left(q_{1}^{*}, q_{2}^{*}\right)=\left[\begin{array}{cc}
1+k \cdot\left(\frac{\partial U_{1}}{\partial q_{1}}+q_{1}^{*} \cdot \frac{\partial^{2} U_{1}}{\partial q_{1}^{2}}\right) & k \cdot q_{1}^{*} \cdot \frac{\partial^{2} U_{1}}{\partial q_{1} \partial q_{2}} \\
k \cdot q_{2}^{*} \cdot \frac{\partial^{2} U_{2}}{\partial q_{2} \partial q_{1}} & 1+k \cdot\left(\frac{\partial U_{2}}{\partial q_{2}}+q_{2}^{*} \cdot \frac{\partial^{2} U_{2}}{\partial q_{2}^{2}}\right)
\end{array}\right]
$$

For the $E_{1}$ the Jacobian matrix is:

$$
\begin{aligned}
J\left(E_{1}\right) & =\left[\begin{array}{cc}
1+k \cdot\left[(1-\mu) \cdot\left(\alpha-b \cdot \ln y_{1}\right)+\mu b\right] & 0 \\
k \cdot q_{2}^{*} \cdot \frac{\partial^{2} U_{2}}{\partial q_{2} \partial q_{1}} & 1+k \cdot q_{2}^{*} \cdot \frac{\partial^{2} U_{2}}{\partial q_{2}^{2}}
\end{array}\right] \\
& =\left[\begin{array}{ll}
A & 0 \\
C & B
\end{array}\right]
\end{aligned}
$$

with $\operatorname{Tr}=A+B$ and $\operatorname{Det}=A B$

From the characteristic equation of $J\left(E_{1}\right)$, we find two eigenvalues:

$$
r_{1}=A \text { and } r_{2}=B
$$


Since

$$
r_{1}=1+k \cdot\left[(1-\mu) \cdot\left(\alpha-b \cdot \ln y_{1}\right)+\mu b\right]
$$

and

$$
(1-\mu) \cdot\left(\alpha-b \cdot \ln y_{1}\right)+\mu b>0
$$

it's clearly seems that $\left|r_{1}\right|>1$ and the $E_{1}$ equilibrium is unstable.

For the $E_{2}$ the Jacobian matrix becomes:

$$
\begin{aligned}
J\left(E_{2}\right) & =\left[\begin{array}{cc}
1+k \cdot q_{1}^{*} \cdot \frac{\partial^{2} U_{1}}{\partial q_{1}^{2}} & k \cdot q_{1}^{*} \cdot \frac{\partial^{2} U_{1}}{\partial q_{1} \partial q_{2}} \\
0 & 1+k \cdot\left[(1-\mu) \cdot\left(\alpha-b \cdot \ln x_{1}\right)+\mu b\right]
\end{array}\right] \\
& =\left[\begin{array}{cc}
D & F \\
0 & E
\end{array}\right]
\end{aligned}
$$

with $T r=D+E$ and $D e t=D E$.

From the characteristic equation of $J\left(E_{2}\right)$, we find two eigenvalues:

$$
r_{1}=D \text { and } r_{2}=E
$$

Since

$$
r_{2}=1+k \cdot\left[(1-\mu) \cdot\left(\alpha-b \cdot \ln x_{1}\right)+\mu b\right]
$$

and

$$
(1-\mu) \cdot\left(\alpha-b \cdot \ln x_{1}\right)+\mu b>0 \text { Eq.(17) }
$$

it's clearly seems that $\left|r_{2}\right|>1$ and the $E_{2}$ equilibrium is unstable. 
For the $E_{*}$ the Jacobian matrix becomes:

$$
\begin{aligned}
J\left(E_{*}\right) & =\left[\begin{array}{cc}
1+k \cdot q^{*} \cdot \frac{\partial^{2} U_{1}}{\partial q_{1}^{2}} \mid\left(q^{*}, q^{*}\right) & k \cdot q^{*} \cdot \frac{\partial^{2} U_{1}}{\partial q_{1} \partial q_{2}} \mid\left(q^{*}, q^{*}\right) \\
k \cdot q^{*} \cdot \frac{\partial^{2} U_{2}}{\partial q_{2} \partial q_{1}} \mid\left(q^{*}, q^{*}\right) & 1+k \cdot q^{*} \cdot \frac{\partial^{2} U_{2}}{\partial q_{2}^{2}} \mid\left(q^{*}, q^{*}\right)
\end{array}\right] \\
= & {\left[\begin{array}{cc}
1-k \cdot(1-\mu) \cdot\left(2 c q^{*}+\frac{3 b}{4}\right) & -\frac{1}{4} k \cdot(1-\mu) \cdot b \\
-\frac{1}{4} k \cdot(1-\mu) \cdot b & 1-k \cdot(1-\mu) \cdot\left(2 c q^{*}+\frac{3 b}{4}\right)
\end{array}\right] } \\
= & {\left[\begin{array}{ll}
G & H \\
H & G
\end{array}\right] }
\end{aligned}
$$

with $\operatorname{Tr}=2 G$ and $\operatorname{Det}=G^{2}-H^{2}$.

From the characteristic equation of $J\left(E_{*}\right)$ we find two eigenvalues:

$$
r_{1}=G+H \text { and } r_{2}=G-H
$$

The $E_{*}$ is locally asymptotically stable if:

$$
\left|r_{i}\right|<1, \quad \forall i=1,2
$$

Taking $\left|r_{1}\right|<1$ the first stability condition is given by:

$$
0<k<\frac{2}{(1-\mu) \cdot\left(2 c q^{*}+b\right)}
$$

and taking $\left|r_{2}\right|<1$ we have the second stability condition which is given by:

$$
0<k<\frac{2}{(1-\mu) \cdot\left(2 c q^{*}+\frac{b}{2}\right)}
$$

As it seems the stability condition of Eq.(25) is the strongest one and this is the total stability condition for the Nash equilibrium $E_{*}$. 


\section{Proposition:}

The Nash equilibrium of the discrete dynamical system Eq.(13) is locally asymptotically stable if

$$
0<k<\frac{2}{(1-\mu) \cdot\left(2 c q^{*}+b\right)}
$$

\section{Numerical Simulations}

\subsection{Focusing on the parameter $\mathbf{k}$ (speed of adjustment)}

In this section we present various numerical results focusing on the parameter $k$, including bifurcation diagrams, Lyapunov numbers and sensitive dependence on initial conditions (Kulenovic, M. and Merino, O. [21]). For this reason we choose to set some fixed values to other parameters as: $\alpha=5, \mathrm{c}=1, \mathrm{~b}=2$ and $\mu=0.2$. By the bifurcation diagrams of the parameter $\mathrm{k}$ against the variables $q_{1}^{*}$ and $q_{2}^{*}$ that are shown in Fig.1 is clear that the equilibrium undergoes a flip bifurcation at $k=0.54$. Then a further increase in speed of adjustment implies that a stable two-period cycle emerges for $0.54<k<0.66$. As long as the parameter $\mathrm{k}$ reduces a four-period cycle, cycles of highly periodicity and a cascade of flip bifurcations that ultimately lead to unpredictable (chaotic) motions are observed when $\mathrm{k}$ is larger than 0.69 .

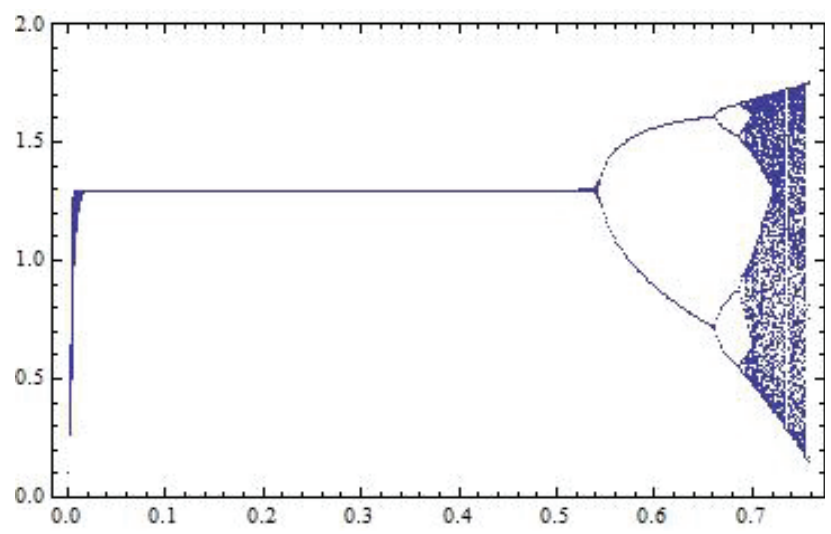

Figure 1: Bifurcation diagrams with respect to the parameter $\mathrm{k}$ against the variables $q_{1}$ and $q_{2}$ with 400 iterations of the map Eq.(13) for $\alpha=5, c=1, b=2$ and $\mu=0.2$.

This unpredictable (chaotic) behavior of the system Eq.(13) is visualized with the useful tool of Lyapunov numbers (Fig.2) (i.e. the natural logarithm of Lyapunov exponents) as a function of the parameter of interest. Figure 2 shows the Lyapunov numbers of the orbit of the system of Eq.(13) for $\alpha=5, c=1, b=2, \mu=0.2$ and $k=0.7$. It is known that if the Lyapunov number is greater than 1 , one has evidence for chaos. 


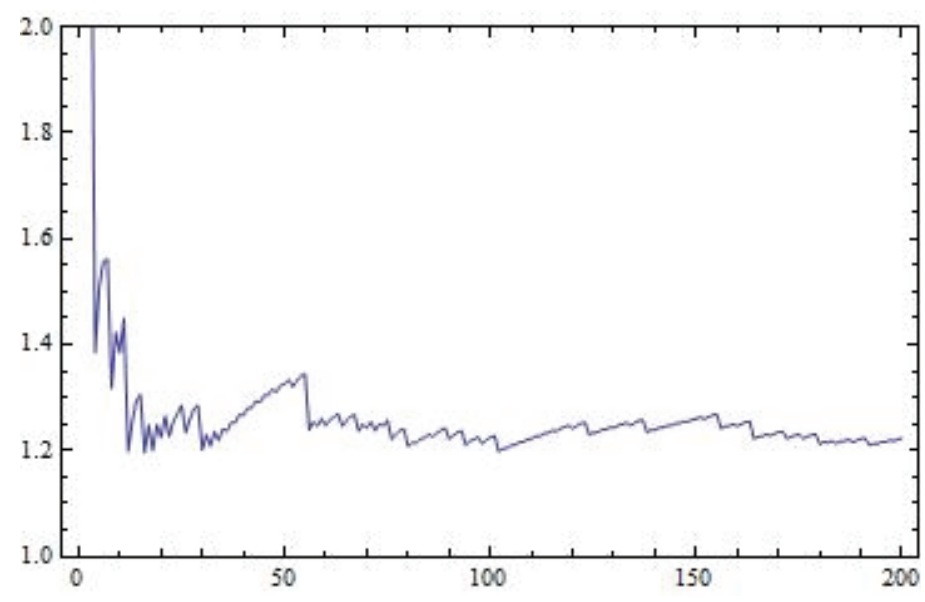

Figure 2: Lyapunov numbers of the orbit of $(0.1,0.1)$ with 8000 iterations of the map Eq.(13) for $\alpha=5, c=$ $1, b=2, \mu=0.2$ and $k=0.7$.

Another characteristic of deterministic chaos is the sensitivity dependence on initial conditions. In order to show the sensitivity dependence on initial conditions of the system Eq.(13), we have computed two orbits with initial points $(0.1,0.1)$ and $(0.101,0.1)$ respectively. Figure 3 shows the sensitivity dependence on initial conditions for $q_{1}-$ coordinate of the two orbits, for the system Eq.(13), plotted against the time with the parameter values $\alpha=5, c=1, d=0.5, \mu=0.7$ and $k=0.58$. At the beginning the time series are indistinguishable; but after a number of iterations, the difference between them builds up rapidly. From these numerical results when all parameters are fixed and only $\mathrm{k}$ is varied the structure of the game becomes complicated through period doubling bifurcations, more complex bounded attractors are created which are aperiodic cycles of higher order or chaotic attractors.
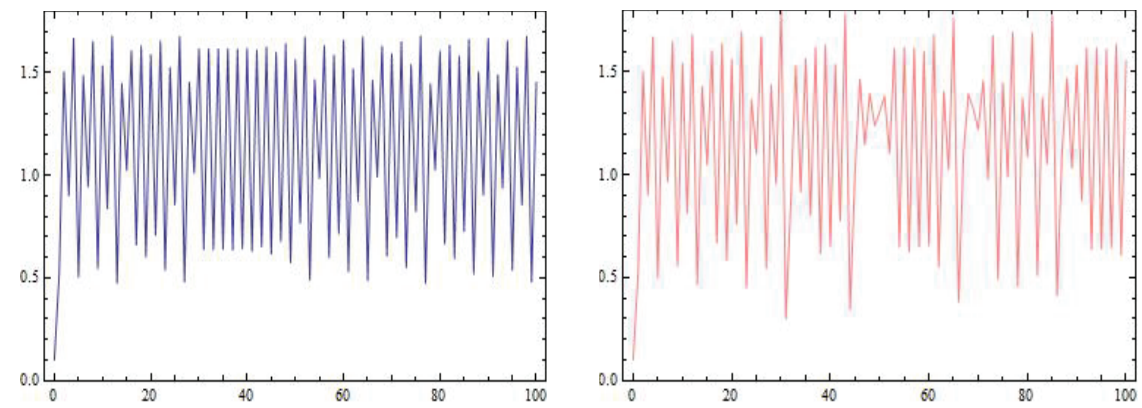

Figure 3: Sensitive dependence on initial conditions for $q_{1}$-coordinate plotted against the time: the orbit of $(0.1,0.1)$ (left) and the orbit of $(0.101,0.1)$ (right) of the system Eq.(13) for $\alpha=5, c=1, b=2, \mu=0.2$ and $k=0.7$.

\subsection{Focusing on the parameter $\mu$ (CS parameter)}

The most interesting parameter of this game is the parameter $\mu$ which reveals the percentage of the consumer surplus CS is taking account by each player. Setting the 
values of the parameters $\alpha=5, c=1, b=2$ and $k=0.7$ a complexity appears for low values of the parameter $\mu$. The Nash equilibrium of the system Eq.(13) is under stability when the parameter $\mu$ takes values greater than 0.48 . Also the differentiation with other studies is that the equilibrium $\left(q^{*}, q^{*}\right)$ is not a fixed point when the parameter $\mu$ is moving into its stability region but it is changing and more specifically the larger the value of $\mu$, the larger the values of $q^{*}$ is taken for the Nash equilibrium. These results are shown by the bifurcation diagrams of Fig. 4 and the chaotic behavior of the system Eq.(13) for $\alpha=5, c=1, b=2, k=0.7$ and $\mu=0.15$ (outside the stability space) is visualized with the Lyapunov numbers of Fig.5. Also, this chaotic behavior has the characteristic sensitivity of this system on its initial conditions for the same values of these parameters (Fig.6).

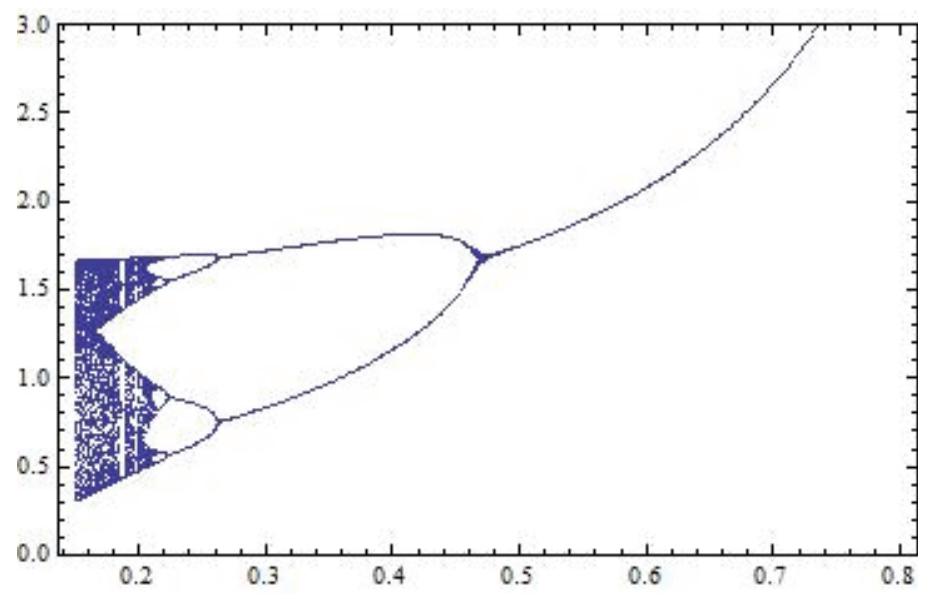

Figure 4: Bifurcation diagrams with respect to the parameter $\mu$ against the variables $q_{1}$ and $q_{2}$ with 400 iterations of the map Eq.(13) for $\alpha=5, c=1, b=2$ and $k=0.7$.

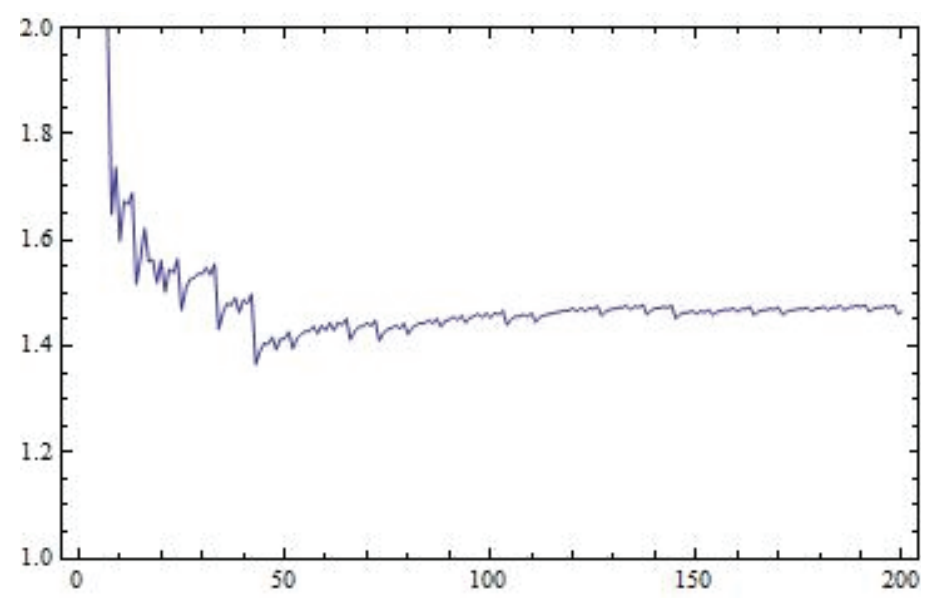

Figure 5: Lyapunov numbers of the orbit of $(0.1,0.1)$ with 2000 iterations of the map Eq.(13) for $\alpha=5, c=$ $1, b=2, k=0.7$ and $\mu=0.15$. 

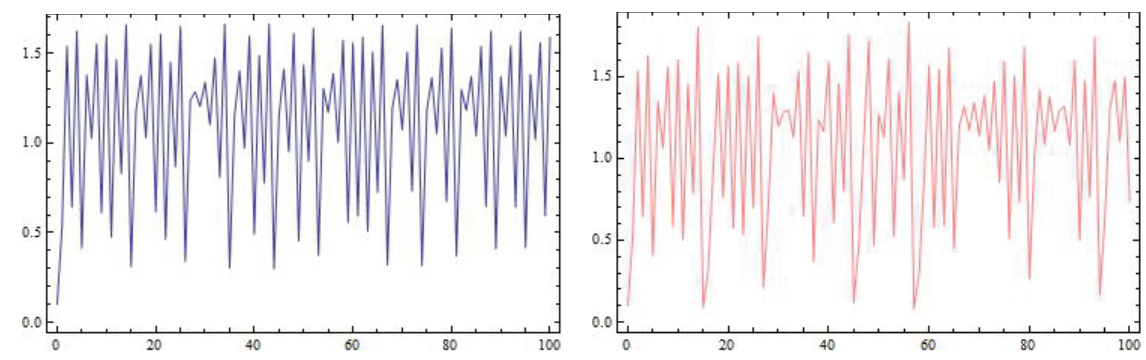

Figure 6: Sensitive dependence on initial conditions for $q_{1}$-coordinate plotted against the time: the orbit of $(0.1,0.1)$ (left) and the orbit of $(0.101,0.1)$ (right) of the system Eq.(13) for $\alpha=5, c=1, b=2, k=0.7$ and $\mu=0.15$.

\section{Conclusions}

The present paper is a partial theoretical approach to our main ongoing research objective, which is to quantify and study an oligopoly of the Greek market. We analyzed through a discrete dynamical system the dynamics of a nonlinear discrete-time duopoly game with a convex and log-linear demand and quadratic cost functions. We suppose that the players not only pursue profit maximization but also take consumer surplus into account. The stability of equilibria, bifurcation and chaotic behavior are investigated. We showed that the speed of adjustment $(k)$ and the parameter of social sensitivity $(\mu)$ may change the stability of equilibrium and cause a structure to behave chaotically. For low values of $\mathrm{k}$ or for high values of $\mu$ the Cournot-Nash equilibrium is stable. Increasing (decreasing) these values, the equilibrium becomes unstable, through period doubling bifurcation.

\section{References}

[1] Agiza H.N., On the analysis of stability, bifurcation, chaos and chaos control of Kopel map, Chaos, Solitons \& Fractals 10, 1909-1916, (1999).

[2] Agiza H.N. and Elsadany A.A., Chaotic dynamics in nonlinear duopoly game with heterogeneous players, Applied Mathematics and Computation 149, 843-860, (2004).

[3] Agiza H.N. and Elsadany A.A., Nonlinear dynamics in the Cournot duopoly game with heterogeneous players, Physica A 320, 512-524, (2003).

[4] Agiza H.N., Hegazi A.S. and Elsadany A.A., Complex dynamics and synchronization of duopoly game with bounded rationality, Mathematics and Computers in Simulation 58, 133-146, (2002).

[5] Agliari A., Gardini L. and Puu T., Some global bifurcations related to the appearance of closed invariant curves, Mathematics and Computers in Simulation 68, 201-219, 
(2005).

[6] Agliari A., Gardini L. and Puu T., Global bifurcations in duopoly when the Cournot point is destabilized via a subcritical Neimark bifurcation, International Game Theory Review 8, 1-20, (2006).

[7] Askar S.S., On complex dynamics of monopoly market, Economic Modelling 31, 586-589, (2013).

[8] Askar S.S., Complex dynamic properties of Cournot duopoly games with convex and logconcave demand function, Operations Research Letters 42, 85-90, (2014).

[9] Baumol W.J. and Quandt R.E., Rules of thumb and optimally imperfect decisions, American Economic Review 54, no. 2, 23-46, (1964).

[10] Bischi G.I. and Kopel M., Equilibrium selection in a nonlinear duopoly game with adaptive expectations, Journal of Economic Behavior and Organization 46, 73-100, (2001).

[11] Bischi G.I., Lamantia F. and Sbragia L., Competition and cooperation in natural resources exploitation: an evolutionary game approach. In: Cararro C., Frangeli V., editors, Game practice and the environment, Cheltenham: Edward Elgar, 187-211, (2004).

[12] Bischi G.I. and Naimzada A., Global analysis of dynamic duopoly game with bounded rationality. In: Filar J.A., Gaitsgory V., Mizukami K., editors, Advances in dynamic games and applications vol. 5, Basel: Birkhauser: 361-385, (1999).

[13] Bischi G.I., Naimzada A.K. and Sbragia L., Oligopoly games with local monopolistic approximation, Journal of Economic Behavior and Organization 46, 73-100, (2001).

[14] Den Haan W.J., The importance of the number of different agents in a heterogeneous asset -- pricing model, Journal of Economic Dynamics and Control 25, 721-746, (2001).

[15] Elaydi S., An Introduction to Difference Equations, $3^{\text {rd }}$ ed., Springer -- Verlag, New York, 2005.

[16] Elsadany A., Awad, Nonlinear Dynamics of Cournot Duopoly Game with Social Welfare, Electronic Journal of Mathematical Analysis and Applications 4, 173-191, (2016).

[17] Fanti L. and Gori L., The dynamics of a differentiated duopoly with quantity competition, Economic Modelling 29, 421-427, (2012).

[18] Gandolfo G., Economic dynamics, Springer, Berlin, 1997.

[19] Hommes C.H., Heterogeneous agent models in economics and finance, In: Tesfatsion L., Judd K.L., editors, Handbook of Computational Economics, Agent - Based Computational Economics vol. 2, Elsevier Science B.V.: 1109-1186, (2006). 
[20] Kopel M., Simple and complex adjustment dynamics in Cournot duopoly models, Chaos, Solitons \& Fractals 12, 2031-2048, (1996).

[21] Kulenovic M. and Merino O., Discreete Dynamical Systems and Difference Equations with Mathematica, Chapman \& Hall/Crc., 2002.

[22] Naimzada A.K. and Ricchiuti G., Complex dynamics in a monopoly with a rule of thumb, Applied Mathematics and Computation 203, 921-925, (2008).

[23] Naimzada A.K. and Sbragia L., Oligopoly games with nonlinear demand and cost functions: two bounded rational adjustment processes, Chaos, Solitons \& Fractals 29, 707-722, (2006).

[24] Puu T., The chaotic monopolist, Chaos, Solitons \& Fractals 5, no.1, 35-44, (1995).

[25] Puu T., The chaotic duopolists revisited, Journal of Economic Bhavior and Organization 37, 385-394, (1998).

[26] Sarafopoulos G., On the dynamics of a duopoly game with differentiated goods, Procedia Economics and Finance 19, 146-153, (2015).

[27] Sarafopoulos G., Complexity in a duopoly game with homogeneous players, convex, log linear demand and quadratic cost functions, Procedia Economics and Finance 33, 358-366, (2015).

[28] Sedaghat H., Nonlinear Difference Equations: Theory with Applications to Social Science Models, Kluwer Academic Publishers (now Springer), 2003.

[29] Tramontana F., Heterogeneous duopoly with isoelastic demand function, Economic Modelling 27, 350-357, (2010).

[30] Westerhoff F., Nonlinear expectation formation, endogenous business cycles and stylized facts, Studies in Nonlinear Dynamics and Econometrics 10, no.4, Article 4, (2006).

[31] Zhang J., Da Q. and Wang Y., Analysis of nonlinear duopoly game with heterogeneous players, Economic Modelling 24, 138-148, (2007).

[32] Zhang J., Da Q. and Wang Y., The dynamics of Bertrand model with bounded rationality, Chaos, Solitons \& Fractals 39, 2048-2055, (2009). 